\title{
Direct imaging of APP proteolysis in living cells
}

\author{
Niccoló Parenti ${ }^{1,2}$, Ambra Del Grosso ${ }^{3}$ \\ Claudia Antoni \\ Marco Cecchini ${ }^{3}$ \\ Pavone $^{1,4}$, Martino Calamai ${ }^{\text {Corresp. }{ }^{1,4}}$ \\ ${ }^{1}$ European Laboratory for Non-linear Spectroscopy (LENS), University of Florence, Sesto Fiorentino, Florence, Italy \\ 3 NEST, Istituto Nanoscienze-CNR and Scuola Normale Superiore, Pisa, Italy \\ 4 National Institute of Optics, National Research Council of Italy (CNR), Florence, Italy \\ Corresponding Author: Martino Calamai \\ Email address: calamai@lens.unifi.it
}

Renato Corradetti $^{2}$, Francesco S

It is now widely accepted that oligomers consisting of $A \beta$ peptide are the cytotoxic species contributing mostly to the development of Alzheimer's disease. A $\beta$ peptide production results from the specific proteolytic processing of the amyloid precursor protein (APP). Deciphering the factors governing the activity of the secretases responsible for the cleavage of APP is still a critical issue. Kits available commercially measure the enzymatic activity of the secretases from cells lysates, in vitro. By contrast, we have developed a prototypal rapid bioassay that provides visible information on the proteolytic processing of APP directly in living cells. APP was fused to a monomeric variant of the green fluorescent protein and a monomeric variant of the red fluorescent protein at the C-terminal and $\mathrm{N}$ terminal, respectively. Changes in the proteolytic processing rate in transfected human neuroblastoma and rat neuronal cells were imaged with confocal microscopy as changes in the red/green fluorescence intensity ratio. The significant decrease in the mean red/green ratio observed in cells over-expressing the $\beta$-secretase BACE1 fused to a monomeric blue fluorescent protein confirms that the proteolytic site is still accessible. Specific siRNA was used to evaluate the contribution of endogenous BACE1. Interestingly, we found that the degree of proteolytic processing of APP is not completely homogeneous within the same single cell, and that there is a high degree of variability between cells of the same type. We were also able to follow with a fluorescence spectrometer the changes in the red emission intensity of the extracellular medium when BACE1 was overexpressed. This represents a complementary approach to fluorescence microscopy for rapidly detecting changes in the proteolytic processing of APP in real time. Moreover, we obtained a quantitatively robust estimate of the changes in the red/green ratio for the above conditions by using a flow cytometer able to simultaneously excite and measure the red and green fluorescence. Our novel approach lay the foundation for a bioassay suitable to study the effect of drugs or particular conditions, to investigate in an unbiased way the the 
proteolytic processing of APP in single living cells in order, and to elucidate the causes of the variability and the factors driving the processing of APP. 


\section{Direct imaging of APP proteolysis in living cells}

3 Niccoló Parenti ${ }^{1,2}$, Ambra Del Grosso ${ }^{3}$, Claudia Antoni ${ }^{1}$, Marco Cecchini $^{3}$, Renato Corradetti ${ }^{2}$,

4 Francesco S. Pavone ${ }^{1,4}$, Martino Calamai* ${ }^{\star 1,4}$

5 'European Laboratory for Non-linear Spectroscopy (LENS), University of Florence, Florence,

6 Italy 50019

7 2Department of Neuroscience, Psychology, Drug Research and Child Health, University of

8 Florence, Florence, Italy

$9{ }^{3}$ NEST, Istituto Nanoscienze-CNR and Scuola Normale Superiore, Piazza San Silvestro 12,

Pisa 56127, Italy.

${ }^{4}$ National Institute of Optics, National Research Council of Italy (CNR), Largo Fermi 6, 50125,

Florence, Italy

${ }^{*}$ Corresponding author

E-mail: calamai@lens.unifi.it 
Alzheimer's disease is a multifactorial disorder caused by the interaction of genetic, epigenetic and environmental factors. The formation of cytotoxic oligomers consisting of $A \beta$ peptide is widely accepted as being one of the main key events triggering the development of Alzheimer's disease. $A \beta$ peptide production results from the specific proteolytic processing of the amyloid precursor protein (APP). Deciphering the factors governing the activity of the secretases responsible for the cleavage of APP is still a critical issue. Kits available commercially measure the enzymatic activity of the secretases from cells lysates, in vitro. By contrast, we have developed a prototypal rapid bioassay that provides visible information on the proteolytic processing of APP directly in living cells. APP was fused to a monomeric variant of the green fluorescent protein and a monomeric variant of the red fluorescent protein at the C-terminal and $\mathrm{N}$-terminal (mChAPPmGFP), respectively. Changes in the proteolytic processing rate in transfected human neuroblastoma and rat neuronal cells were imaged with confocal microscopy as changes in the red/green fluorescence intensity ratio. The significant decrease in the mean red/green ratio observed in cells over-expressing the $\beta$-secretase BACE1, or the $\alpha$-secretase ADAM10, fused to a monomeric blue fluorescent protein confirms that the proteolytic site is still accessible. Interestingly, we found that the degree of proteolytic processing of APP is not completely homogeneous within the same single cell, and that there is a high degree of variability between cells of the same type. We were also able to follow with a fluorescence spectrometer the changes in the red emission intensity of the extracellular medium when BACE1 was overexpressed. This represents a complementary approach to fluorescence microscopy for rapidly detecting changes in the proteolytic processing of APP in real time. In order to allow the discrimination between the $\alpha$ - and the $\beta$-secretase activity, we have created a variant of mChAPPmGFP with a mutation that inhibits the $\alpha$-secretase cleavage without perturbing the $\beta$-secretase processing. Moreover, we obtained a quantitatively robust estimate 
51 of the changes in the red/green ratio for all the above conditions by using a flow cytometer able

52 to simultaneously excite and measure the red and green fluorescence. Our novel approach lays

53 the foundation for a bioassay suitable to study the effect of drugs or particular conditions, to

54 investigate in an unbiased way the proteolytic processing of APP in single living cells, and to

55 elucidate the causes of the variability and the factors driving the processing of APP.

56

57

58

59

60

61

62

63

64

65

66

67

68

69 


\section{Introduction}

71 In the last decades the incidence of Alzheimer's disease (AD) has risen due to an increase in

72 the lifespan, representing more and more a serious social problem. The first steps in the

73

74

75

76 pathological cascade involve protein quality control impairment and the failure of misfolded proteins clearance mechanisms, with subsequent accumulation and oligomeritazion of neurotoxic amyloid- $\beta$ peptide. $A \beta$ oligomers decrease the synaptic efficacy, activate microglia and astrocytes causing an inflammatory response, alter the degree of tau's phosphorylation leading to tangles, deposit in the brain blood vessels and cause neuronal loss and neurotransmitter deficits(Selkoe and Hardy, 2016). The generation of amyloid- $\beta$ peptide results from the sequential cleavage of the amyloid precursor protein (APP) by $\beta$-secretase and $\gamma$ secretase(Haass, 2004) (De Strooper, 2010). This process is in competition with an alternative non-pathogenic pathway lead by the $\alpha$-secretase(De Strooper, 2010). APP is a plasma membrane glycoprotein whose original function remains unclear (Senechal, Larmet and Dev, 2006). The $\beta$-secretase and the other secretases have become obvious therapeutic targets for drug research in AD (MacLeod, et al., 2015). Several strategies have mainly focused on the inhibition of $\beta$-secretase and $\gamma$-secretase or, alternatively, on $A \beta$ clearance. Unfortunately, none of these pharmacological therapies has yet passed the phase II/III trial due to a lack of encouraging results, or to the onset of adverse side effects (MacLeod, et al., 2015). Despite the recent failures, significant hope still lies around the secretase targeting approach. With this regard, the full understanding of the factors governing the rate of proteolytic processing of APP is imperative. Commercial kits (Sigma-Aldrich, Abcam) to evaluate the activity of the $\beta$ secretase BACE1 from human and animal samples are available. These assays use a specific BACE1 substrate conjugated to two reporter molecules. In the uncleaved form, the fluorescence of the reporter is quenched by the physical proximity of the other molecule. After the cleavage by $\beta$-secretase, the two molecules are separated and a fluorescent signal can be acquired with 
95

96

98 intensity proportional to the enzymatic activity. This type of kit is very efficient in detecting the activity of BACE1 from purified samples, but remains an in vitro evaluation. By contrast, we have developed a consistent bioassay to quantify in an unbiased way the the proteolytic processing of APP in single living cells. The key element of our assay is a chimeric construct of APP with two different fluorescent proteins fused at the opposite terminals. Changes in the proteolytic processing of APP are seen as changes in the intensity ratio of the two fluorescent protein tags. We demonstrate that our mChAPPmGFP construct is efficiently recognized and cleaved by both $\alpha$ - and $\beta$-secretase. The activity of each individual secretase can be discriminated by using specific siRNAs or, alternatively, by introducing specific mutations at the cleavage sites of APP. Here, we have created a mutated variant of mChAPPmGFP that can be cleaved almost exclusively by $\beta$-secretase (mChAPPP1 mGFP). The mutation, a Lys to Val substitution at position 612 of APP695, just before the $\alpha$-secretase cleavage site (this position is called P1), has already been used to verify the sequence specificity of $\alpha$-secretase cleavage(Sisodia, 1992). Sisodia tested a series of mutants at the P1 position, and found by immunoprecipitation studies that only one, the Lys to Val substitution, prevents the a-secretase cleavage.

Finally, we obtained a quantitatively robust validation of our bioassay by using a flow cytometer able to simultaneously excite and measure the emission intensity of the two fluorescent proteins. 


\section{Materials and Methods}

\section{Cloning}

118 To generate the fusion constructs of mChAPPmGFP and mChAPP770mGFP we used

119 APP695 and APP770 contained in the vector pCMV-AC-GFP (Origene, Rockville MD, USA).

120 First, we inserted new restriction sites (Nhel, Ascl) after the signal peptide using the GeneArt

121 Site-Directed Mutagenesis Kit (ThermoFisher Scientific), using

GCCGCCTGGACGGCTCGGGCGGCTAGCCAGGGCGCGCCTCTGGAGGTACCCACTGATG

$\mathrm{G}$ as forward primer, and

CCATCAGTGGGTACCTCCAGAGGCGCGCCCTGGCTAGCCGCCCGAGCCGTCCAGGCGGC

as reverse primer. The mCherry sequence was amplified from its original plasmid (pmCherry-C1 vector, Clontech) with a PCR reaction (forward primer:

TAAGCAGCTAGCATGGTGAGCAAGGGCGAGGAG, reverse primer

TGGCGCGCCTGTTCCACGACTCTTGTACAGCTCGTCCATG) adding the restriction sequences for the enzymes Nhel and Ascl. After digestion, the sequence of mCherry was inserted at the N-term of APP. In order to substitute the dimerizing TurboGFP present in the pCMV-AC-GFP vector with the monomeric green fluorescent protein mTagGFP2, mCherryAPP695 and mCherry-APP770 were excised from their original plasmid using the restriction enzymes Sgfl and Notl and inserted in the plasmid pCMV6-AN-mGFP (commercial name of mTagGFP2, Origene) to obtain the final fusion constructs mCherry-APP695-mGFP and mCherry-APP770-mGFP. The QuikChange Lightning Site-Directed Mutagenesis Kit (Agilent) was used to mutagenize the mChAPPmGFP construct in order to create a new plasmid

137 (mChAPPP1mGFP ) carrying the P1 mutation, a Lys to Val substitution at P1 position (amino acid 612 of APP695), that is the amino acid located before the $\alpha$-secretase cleavage site(Sisodia, 1992) (forward primer: GAAGTTCATCATCAAGTATTGGTGTTCTTTGC, reverse 
primer: GCAAAGAACACCAATACTTGATGATGAACTTC).

141 To generate the fusion construct Bace1-mBFP and HA-Bace1-mBFP, we started from Bace1

142 contained in the vector pCMV6-ENTRY (NM_012104, Origene, Rockville, MD, USA). We

143 opened the vector using the restriction enzymes Notl and Sacll. mBFP (original name

144 mTagBFP2) was extracted with a PCR reaction from the plasmid pmTagBFP2-N1(Subach, et

145 al., 2011) (forward primer: TAAGCAGCGGCCGCGAATGAGCGAGCTGATTAAGG, reverse

146 primer: CCGAGTCCGCGGTTAATTAAGCTTGTGCCCCAG) to add the restriction sequences of

147 the enzymes Notl and Sacll. After digestion, the sequence of mBFP was inserted in the plasmid

148 of Bace1 previously opened with the same enzymes, to obtain the final fusion construct Bace1-

149 mBFP. With a site-directed mutagenesis reaction (ThermoFisher Scientific) we added the

150 restriction sites for Nhel and Ascl at the N-term of Bace1-mBFP (forward primer:

GGAGTGCTGCCTGCCCACGGCGCTAGCCAGGGCGCGCCtACCCAGCACGGCATCCGGCT

$\mathrm{G}$, reverse primer

CAGCCGGATGCCGTGCTGGGTAGGCGCGCCCTGGCTAGCGCCGTGGGCAGGCAGCACT

CC). We opened the plasmid of Bace1-mBFP and inserted the annealed oligos containing the sequence for the HA tag (forward oligo: CTAGCTATCCGTACGACGTACCAGACTACGCAGG, reverse oligo: CGCGCCTGCGTAGTCTGGTACGTCGTACGGATAG). After ligation we obtained the plasmid of the construct HA-Bace1-mBFP. ADAM10mBFP was created by the fusion of the mTagBFP2 gene downstream of the ADAM10 gene contained in the pRK5M-ADAM10 construct (Addgene). The final part of the sequence coding for ADAM10 was mutagenized in order to introduce two new restriction sites, unique within the entire sequence of the plasmid. The site-directed mutagenesis was performed with the QuikChange Lightning Site-Directed Mutagenesis Kit (Agilent Technologies), using GAGGACCTGCTGCGTACGGTCGCGCGCGCTTGGCCGCCA as forward primer and TGGCGGCCAAGCGCGCGCGACCGTACGCAGCAGGTCCTC as reverse primer, containing the restriction sequences for the BsiWI e BssHII enzymes. The mTagBFP2 sequence was 
166 amplified from its original plasmid (pmTagBFP2-N1, Addgene) with a PCR reaction (forward

167 primer GAGCGTACGATGAGCGAGCTGATTAAGGAG, reverse primer

168 GTAGCGCGCTTAATTAAGCTTGTGCCCCAG, the forward primer containing the BsiWI

169 restriction site and the reverse the BssHII one). After digestion, the sequence of BFP2 was

170 inserted at the C-terminal of ADAM10.

171 apAPPha was created from mChAPPmGFP using the restriction sites for Nhel and Ascl at the

172 N-term. First, we opened the plasmid with these enzymes and inserted the annealed oligos

173 containing the sequence for the genetic tag AP (forward oligo:

174 CTAGCGAGGGCCTGAACGATATCTTCGAGGCCCAGAAGATCGAGTGGCACGAGAGTGGre

175 verse oligo:

CGCGCCACTCTCGTGCCACTCGATCTTCTGGGCCTCGAAGATATCGTTCAGGCCCTCG).

177 Afterwards, we opened the plasmid with the restriction enzimes Notl and Sacll in order to

178 substitute mGFP with the annealed oligos containing the sequence for the genetic tag HA

179 (forward oligo:

GGCCGCTCGAGTATCCGTACGACGTACCAGACTACGCAGTTTAAACCCGC,

reverse oligo: GGGTTTAAACTGCGTAGTCTGGTACGTCGTACGGATACTCGAGC).

\section{Cell cultures and transfection}

Human SH-SY5Y neuroblastoma cells (A.T.C.C. Manassas, VA, USA) were cultured in Dulbecco's Modified Eagle's Medium (DMEM) F-12 supplemented with 10\% FBS, 1\% penicillin/streptomycin solution. HEK cells were cultured in DMEM Glutamax supplemented with $10 \%$ FBS, 1\% penicillin/streptomycin solution. Primary rat hippocampal neurons were obtained after dissociation of hippocampi purchased from Brainbits, UK. Neurons were plated on coverslips coated with poly-L-ornithine and grown in Neurobasal medium supplemented with $2 \%$ B27, 0,5 mM glutamine, 12,5 mM glutamate and 0,1\% penicillin/streptomycin. All the cell types were cultured in a humidified chamber at $5 \% \mathrm{CO}_{2}$ and $37^{\circ} \mathrm{C}$. Neuroblastoma SH-SY $5 \mathrm{Y}$ cells 
191 were transfected with Fugene HD (Promega, USA) 24-48 h before imaging. HEK cells were

192 transfected with Lipofectamine 2000 (ThermoFisher Scientific) $24-48 \mathrm{~h}$ before imaging. To

193 transfect hippocampal neurons DNA was mixed with Lipofectamine 2000 in $0,5 \mathrm{ml}$ of

194 Neurobasal supplemented with $0,5 \mathrm{mM}$ glutamine at $37^{\circ} \mathrm{C}$ in $5 \% \mathrm{CO}_{2}$ for $60 \mathrm{~min}$. Afterwards

195 neurons were rinsed with Neurobasal, transferred to the original medium at $37{ }^{\circ} \mathrm{C} 5 \% \mathrm{CO}_{2}$ and

196 imaged after 24-48 h. apAPPha was co-transected with BirA, a biotin ligase that specifically

197 biotinylates the AP tag . Biotin was added to the cell medium (final concentration $10 \mu \mathrm{M}$ ) 8 hours

198 after transfection and 12 hours before imaging(Howarth, et al., 2005). siRNA Silencer®

199 Validated siRNA against BACE1 (105154) and Silencer $®$ Negative Control siRNA were 200 purchased from ThermoFisher. Supplementary Figure S5 shows the silencing efficiency of 201 Bace1-siRNA.

\section{Cell imaging}

203

204

205

206

207

208

209

210

211

212

213

214

215

Live cell imaging was performed on a Nikon Eclipse TE300 C2 LSCM (Nikon, Japan) equipped with a Nikon 60x immersion oil objective (Apo Plan, NA 1.4). Cells were growth on $18 \mathrm{~mm}$ coverslips and mounted on a custom chamber containing $1 \mathrm{ml}$ of Leibovitz's medium (ThermoFisher). For immunolabelling, cells were fixed with $4 \%$ PFA, rinsed with $\mathrm{PBS}\left(+\mathrm{MgCl}_{2}\right.$ $0,5 \mathrm{mM},+\mathrm{CaCl}_{2}$ 0,8 mM) and permeabilized with 0,075\% Triton X. After rinsing with PBS and blocking with 4\% BSA PBS, cells were labelled with primary (1:1000 rabbit anti-HA ab9110 Abcam, UK-) and secondary antibodies (goat anti-rabbit Alexa 488,--ThermoFisher, diluted at 1:500), or streptavidin Alexa 568 (ThermoFisher diluted at 1:500), diluted in PBS with 4\% BSA. After rinsing again with PBS, coverslips were mounted on a glass slide and imaged with LSCM, equipped with Coherent CUBE (diode 405 nm), Melles Griot (Argon 488 nm) and Coherent Sapphire (Sapphire $561 \mathrm{~nm}$ ) lasers. Emission filters for imaging were 452/45 nm, 514/30 nm and 595/60 nm. For surface labeling, living SH-SY5Y cells were growth on $18 \mathrm{~mm}$ coverslips, rinsed with PBS and incubated with primary antibodies (1:100 rabbit anti-mCherry ab167453, or 
216 1:1000 rabbit anti-HA ab9110 -Abcam, UK-) diluted in PBS with 4\% BSA for 30 minutes on ice,

217 to inhibit endocytic events. After rinsing with PBS,cells were incubated with secondary

218 antibodies (1:500 goat anti-rabbit Alexa 405) diluted in PBS with 4\%BSA for 15 minutes. After

219 rinsing again the coverslips, cells were mounted on a custom chamber filled with Leibovitz's

220 medium and imaged with the same LSCM configuration mentioned above.

\section{Western blotting}

222 Human SH-SY5Y neuroblastoma cells were cultured in 6 wells plates. $24 \mathrm{~h}$ after transfection,

223 the extracellular medium was recovered and the cells were lysed using $300 \mu$ l of PBS and

224 Laemmli buffer. Lysed cells were collected in safe lock 1,5 ml tubes and immersed in boiling

225 water for 5 minutes, then sonicated two times for 15 seconds and boiled again for 5 minutes.

226 The recovered medium was centrifuged at $10000 \mathrm{rpm}$ for $5 \mathrm{~min}$ in order to remove cell debrees

227 and other impurity, and then treated as the cell lysate. Samples were loaded in pre-casted

228 polyacrylamide gels (ThermoFisher Scientific, MA USA) and blotted on PVDF membranes

229 (ThermoFisher Sicnetific, MA USA). Membranes were blocked in 5\% milk in PBST overnight at $2304^{\circ} \mathrm{C}$ or $1 \mathrm{~h}$ at room temperature. After blocking, membranes were rinsed three times with PBST

231 and incubated at room temperature with primary antibody (in PBST with 4\% BSA) for $1 \mathrm{~h}$ and for

232 an additional hour with secondary anti-rabbit antibody conjugated to horseradish peroxidase

233 (1:20000). The primary antibodies were: rabbit anti-mCherry (1:1000, Abcam, UK, ab167453),

234 rabbit anti-APP (1:200, Abcam ab15272), rabbit anti-vimentin (1:2500, Abcam ab92547

235 EPR3776) mouse anti-aß (1:100, Abcam ab11132 DE2b4), mouse anti-GFP (1:1000 Abcam,

236 ab291 LGB-1). Membranes were rinsed with the HRP substrate Pico Dura (ThermoFisher

237 Scientific, MA USA) and imaged after 5 min with a Chemidoc MP system (Bio-Rad, CA USA). In

238 order to perform the stripping procedure, the blotted PVDF membranes were covered with

239 Seppro Stripping Buffer (Sigma Aldrich, MO USA) and incubated for 5-10 min at room

240 temperature. The stripping buffer was then discarded and the incubation was repeated with 
241 fresh Seppro buffer for 5-10 min. After discarding the buffer, membranes were washed for two

242 times with PBS for 10 minutes, then washed for two times with TBST for 5 minutes and blocked.

\section{Fluorescence spectroscopy}

244 Fluorescence spectra from $585 \mathrm{~nm}$ to $700 \mathrm{~nm}$ were acquired at $25^{\circ} \mathrm{C}$ in a $10 \times 10 \mathrm{~mm}$ quartz

245 cuvette using a Perkin-EImer LS 55 spectrofluorimeter (Waltham, MA, USA) equipped with a

246 thermostated cell holder attached to a Haake F8 water bath (Karlsruhe, Germany). Excitation

247 wavelength was $550 \mathrm{~nm}$.

\section{FACS}

249 Cells were harvested after mild trypsin treatment and centrifuged for $5 \mathrm{~min}$ at $330 \mathrm{~g}$; the

250

251

252

253

254

255

256

257

258

259

260

261

262

263 obtained pellets were washed and re-suspended in PBS. We cannot exclude that the treatment with trypsin might affect the integrity of the ectodomain of mChAPPmGFP; however, all the conditions of transfection tested would have been affected equally without influencing the conclusion of our experiments. Flow cytometry was performed by using a S3 flow cytometer (Bio-Rad) equipped with 488 and $561 \mathrm{~nm}$ diode-pumped solid-state lasers. mGFP was excited using the $488 \mathrm{~nm}$ laser, and the emitted fluorescence was collected through a 586/25 nm bandpass filter; mCherry was excited with the $561 \mathrm{~nm}$ laser and its fluorescence was collected through a 615/25 nm band-pass filter. Data were analysed using ProSort software (Bio-Rad), by dividing the scatter plot of mGFP versus mCherry into two equally populated regions under control conditions. The cellular debris was excluded from the quantification by gating cells on the Forward scatter area/Side scatter area (FSC/SSC) graph, while doublets were excluded by gating the cells on the Forward scatter height/ Forward scatter area (FSC/FSC) graph; at least 10000 events were acquired for each sample. 


\section{Results}

\section{5 mChAPPmGFP is correctly targeted to the plasma}

\section{6 membrane}

267

268

269

270

271

272

273

274

275

276

277

278

279

280

281

282

283

284

285

APP695, the isoform mainly expressed in the central nervous system (Nalivaeva and Turner, 2013), was fused to a monomeric variant of the green fluorescent protein (mGFP) at the Cterminal and to a monomeric red fluorescent protein (mCherry) at the N-terminal (Fig. 1). In this way the extremities of APP situated at the opposite sides of the plasma membrane are labeled with two different fluorescent proteins. Once the N-term mCherry containing domain is cleaved, the fluorescence intensity ratio between the red and green signals changes. It is thus possible to monitor in living cells a higher or lower processing of APP in function of the ratio shift. From herein, we will refer to this chimeric construct bearing the APP695 isoform as to mChAPPmGFP. The same procedure was repeated to obtain the fusion construct of APP770 (mChAPP770mGFP), an alternative isoform expressed more ubiquitously (Zheng and Koo, 2011).

mCherry was fused immediately after the signal peptide, which is necessary to target APP to the plasma membrane, and away from the site recognized and cleaved by the $\beta$-secretase. In order to test if mChAPPmGFP is correctly targeted to the plasma membrane, we performed a surface immunolabelling of transfected human SH-SY5Y neuroblastoma cells with a primary anti-mCherry antibody (Fig. 2a). Confocal laser scanning microscopy (CLSM) imaging reveals a good degree of labeling only in cells expressing mChAPPmGFP, confirming that mCherry is correctly folded and present on the surface of the cell. We observed red clusters not colocalising with anti-mCherry, thus indicating their intracellular localization. 
286 To confirm that the full-length chimeric mChAPPmGFP protein was expressed, we examined

287 the colocalization of mCherry and mGFP in neuroblastoma cells (Fig. 2 b-d) and rat

288 hippocampal neurons (Fig. 2 e-g). The toolbox available with the JACoP plug-in(Bolte and

289 Cordelieres, 2006) under the ImageJ software(Schneider, Rasband and Eliceiri, 2012) was used

290 to quantify the degree of colocalization. We found that in most cells the plot of the pixel intensity

291 of the green against that of the red channel (scatterplot) follows a linear relationship, with an

292 average Pearson's coefficient (an estimate of the quality of the linear relationship between the

293 two signals with 1 standing for complete positive correlation) of 0.91 . We also found an average

294 Manders' overlap coefficient(Manders, et al., 1992) M1 (indicating the fraction of mCherry

295 overlapping with mGFP) of 0.97 and an M2 (fraction of mGFP overlapping mCherry) of 0.85.

296 The measured Costes' randomization P-value(Costes, et al., 2004) of $100 \%$ excludes that the

297 co-localization of pixels is due to chance. An additional method such as Li's intensity correlation

298 analysis(Li, et al., 2004) further supports a very good degree of colocalization, indicating an

299 abundant presence of full-length mChAPPmGFP. We recurrently observed small red fluorescent

300 punctae, at which did not correspond a comparable level of green fluorescence intensity. Similar

301 data were obtained for mChAPP770mGFP (Supplementary Fig. S1).

302 Due to their size and consequent steric hindrance, the two fluorescent tags could alter the

303 distribution features of APP. However, we did not observe any significant difference in the

304 overall cellular distribution after immunolabeling of an overexpressed construct of APP695

305 bearing two small tags (AP(Howarth, et al., 2005) and HA(Schembri, et al., 2007), apAPPha) at

306 the two opposite terminals (Fig. 2h-j). As previously reported(Collins, et al., 2010;Guo, et al.,

307 2012; Haass, et al., 2012), a large fraction of intracellular mChAPPmGFP is localized at the level

308 of the Golgi apparatus, in the perinuclear region (Supplementary Fig. S2). In agreement with an

309 elegant study investigating the subcellular localization of endogenous full length APP in

310 neurons(Guo, et al., 2012), mChAPPmGFP also shows partial co-localization with the 
311 endoplasmic reticulum, but very little with early endosomes and lysosomes (Supplementary Fig.

312 S2). We find this study of particular relevance since it shows with a knock-out model that many

313 antibodies against APP commonly used for immunofluorescence experiments give rise to non-

314 specific labeling.

\section{Fluorescence intensity ratio heterogeneity}

316 We then measured the ratio between the red and the green fluorescence intensity in

317 neuroblastoma cells (Fig. 3 a) and rat hippocampal neurons (Fig. 3 b). The ratio image was

318 generated using the Ratio Plus available on ImageJ (Schneider, et al., 2012). Regions of

319 interest (ROIs) for the calculation of the mean ratio value per cell were chosen by using a

320 constant threshold value on the maximum projection of mGFP stacks. The ROls were then

321 applied to the corresponding ratio image. The gain used for the acquisition of neuronal images

322 was different from that of neuroblastoma cells due to differences in the level of protein

323 expression. We observed a certain degree of intracellular heterogeneity in the red/green ratio,

324 with lower red/green ratio in the peripheral areas of the cells. In addition, we observed a

325 relatively high intercellular variability among the same population of transfected neuronal or

326 neuroblastoma cells.

\section{8 mChAPPmGFP is cleavable by Bace1}

329 In order to check if our fusion protein was correctly folded, recognized and cleaved, we co-

330 transfected SH-SY5Y (Fig. 4) and neuronal cells (Fig. 5) with mChAPPmGFP together with $\beta$ -

331 secretase Bace1 fused with mTagBFP2 (mBFP) at the C-term. We found that overexpressed

332 BACE1 is aboundantly present on the plasma membrane (Supplementary Fig. S3). When

333 Bace1-mBFP cleaves mChAPPmGFP on the cell surface, the soluble fragment mCherry-sAPP $\beta$

334 is released in the extracellular medium. We compared the mean red/green ratio in cells 
335

336

337

338

339

340

341

342

343

344

345

346

347

348

349

350

351

352

353

354

355

356

357

358

359

expressing mChAPPmGFP with or without Bace1-mBFP (Fig. 4 a-c; Fig. 5). While we did not find statistically significant differences in cells transfected with siRNA against Bace1 (although an unpredicted minor reduction in the ratio is visible), the overexpression of Bace1-mBFP dramatically decreased the ratio. Although APP might undergo a major processing by $\alpha-$ secretase under physiological conditions, the contribution of the latter is negligible when APP is overexpressed with BACE1. Cells transfected with mChAPP770mGFP showed a comparable trend.

Equivalent results were obtained when performing western blots of both cell lysate and extracellular medium of SH-SY5Y cells co-transfected with mChAPPmGFP and Bace1-mBFP or Bace1-siRNA, using anti-mCherry as primary antibody for chemoluminescent detection (Fig. 4d). In the lysate of cells co-transfected with mChAPPmGFP and Bace1-siRNA, two bands were detected, one more intense at $152 \mathrm{KDa}$, corresponding to full-length mChAPPmGFP (expected molecular weight $134 \mathrm{KDa}$ ), and one at $125 \mathrm{KDa}$, possibly corresponding to mCherry-sAPPa (expected molecular weight $100 \mathrm{KDa}$ ) generated by endogenous $\alpha$-secretase. For cells cotransfected with mChAPPmGFP and Bace1-mBFP, the same two bands were visible, but in this case the lower band was more intense, due to an increased production of mCherry-sAPP $\beta$, which has a molecular weight (expected molecular weight $94 \mathrm{KDa}$ ) comparable to mCherrysAPPa. Blotting of the extracellular medium revealed only one band at $121 \mathrm{KDa}$, corresponding to the released mCherry-sAPPfragment. A considerably higher intensity band was found in the case of cells co-transfected with Bace1 (increased production of mCherry-sAPP $\beta$ ) as compared to those co-transfected with Bace1-siRNA (mostly mCherry-sAPPa). Analogous results were obtained for the cell lysate and the extracellular medium of cells transfected with mChAPP770mGFP, with the bands being at higher molecular weights: $163 \mathrm{KDa}$ for the uncleaved mChAPP770mGFP (expected molecular weight $142 \mathrm{KDa}$ ) and $138 \mathrm{KDa}$ for the mCherry-sAPP770ß (expected molecular weight $102 \mathrm{KDa}$ ). 
360 Membrane stripping and reprobing with anti-GFP, anti-APP (directed against the N-terminal 44-

36162 amino acids) or anti-A $\beta$ (directed against amino acids 1-17 of $A \beta$ ) generated analogous

362 results. At long time exposures of the blot, bands corresponding to the C-terminal fragments of 363 mChAPPmGFP (C83/89-mGFP and AICD-mGFP) are visible (Supplementary Fig. S4). These

364 data corroborate further that our construct is recognized and processed by BACE1.

365 Furthermore, we collected and analyzed the supernatant media from different transfection

366 conditions of SH-SY5Y cells with a fluorescence spectrophotometer. In agreement with the

367 above results, we observed higher fluorescence intensity at $610 \mathrm{~nm}$ (maximum emission peak

368 for mCherry) in cells co-expressing mChAPPmGFP together with Bace1-mBFP (Fig. 4 e). This

369 result further supports that $\beta$-secretase recognizes and cleaves our fusion APP construct, which

370 is then in part released in the extracellular medium as mCherry-sAPP $\beta$. Similar results were

371 found in HEK cells (Supplementary Fig. S5). Our data show an unexpected slight increase in

372 fluorescence intensity at $610 \mathrm{~nm}$ in the cells co-transfected with Bace1-siRNA compared to the

373 control condition. Overall these results suggest that the site cleaved by $\beta$-secretase is still

374 accessible and that is possible to follow the processing of APP as function of the ratio shift.

375 We finally validated our results with FACS flow cytometry, a statistically robust technique

376 capable of analyzing the red and green fluorescence intensity for a large number of single cells.

377 SH-SY5Y cells were co-transfected with mChAPPmGFP and Bace1-mBFP, or control siRNA, or

378 Bace1-siRNA. Two different areas in the red versus green fluorescence scatter plot were

379 arbitrarily chosen so that both areas contained the same number of cells co-transfected with

380 mChAPPmGFP and control siRNA (Fig. 6). The overexpression of Bace1-mBFP changed

381 significantly the ratio between the number of cells in the red and green areas. We observed only

382 a slight, although not significant, increase in the ratio when cells were co-transfected with siRNA

383 directed against Bace1. Comparable results were found using HEK cells (Supplementary Fig. 384 S6). 
387

389

390

391

392

393

394

395

396

397

398

399

400

401

402

403

404

405

406

407

408

SH-SY5Y neuroblastoma cells were co-transfected with mChAPPmGFP or mChAPPP1mGFP and ADAM10mBFP in order to test whether the P1 mutation really inhibits the a-secretase activity. We compared the mean red/green ratio in cells overexpressing ADAM10mBFP and mChAPPmGFP with or without the P1 mutation (Fig. 7a-b). The red/green ratio was found to be significantly higher in cells overexpressing mChAPP ${ }^{\mathrm{P} 1} \mathrm{mGFP}$ compared to mChAPPmGFP, showing not only that the ADAM10mBFP construct is proteolytically active, but also that the presence of the P1 mutation strongly prevents the cleavage by a-secretase. These results were validated by FACS flow cytometry (Fig. 7c and Fig S7). SH-SY5Y cells were co-transfected with mChAPPmGFP or mChAPPP1mGFP and ADAM10mBFP or mBFP. As above, two different areas containing the same number of cells co-transfected with mChAPPmGFP and mBFP were used to gate the scatter plots of cells co-transfected with the other conditions and calculate the mean ratio between the number of cells in the red area over the number of cells in the green area. The number of cells in the red area significantly increased with the overexpression of $\mathrm{mChAPP}^{\mathrm{P}}{ }^{\mathrm{m} G F P}$ compared to the wild-type, both in the presence of ADAM10mBFP or mBFP, denoting an almost complete resistance to $\alpha$-secretase proteolysis. Moreover, SH-SY5Y cells were co-transfected with $\mathrm{mChAPPP} 1 \mathrm{mGFP}$ and $\mathrm{BACE} 1 \mathrm{mBFP}$ in order to verify potential sideeffects of the P1 mutation on the $\beta$-secretase processing. The ratio between the number of cells in the red and green areas resulting from the co-expression of $\mathrm{mChAPP}^{\mathrm{P}} \mathrm{mGFP}$ and BACE1mBFP is comparable with that from mChAPPmGFP and BACE1mBFP. This data suggests that the variant of APP carrying the P1 mutation is hardly processed by ADAM10, while still cleavable by Bace1. 


\section{Discussion}

410 We have successfully demonstrated the proof of principle of a bioassay to evaluate the

411 processing of APP directly in single living cells, a less biased method compared to the in vitro

412 kits that are commercially available. To estimate the rate of processing of APP, we measured

413 the variation in the red/green fluorescence ratio emitted from cells overexpressing

414 mChAPPmGFP. We demonstrated that mChAPPmGFP is correctly folded and targeted to the

415 plasma membrane, and it is still cleavable by the $\beta$-secretase Bace1. We have noticed that a

416 similar approach has been tested in two previous works(Coughlan, et al., 2013;Villegas,

417 Muresan and Ladescu Muresan, 2014). In one of these papers, the residual C-terminal region of

418 APP770 (106 amino acids) carrying the Swedish mutation was fused with two different

419 fluorescent proteins (DsRed2 and EGFP) at the opposite terminal sides(Coughlan, et al., 2013).

420 By contrast, our mChAPPmGFP construct is generated from the full length APP695 and

421 contains the original signal-peptide sequence that is required for targeting the newly

422 synthesized protein to the plasma membrane. Furthermore, in order to avoid oligomerization

423 events, we used two monomeric fluorescent proteins, mCherry and mGFP. These two

424 fluorescent proteins have comparable maturation times of approximately 10-15 minutes(Shaner,

425 et al., 2004;Subach, et al., 2008), thus limiting the rise of artifacts due to different maturation

426 events. In agreement with the fact that the mCherry and the mGFP tags are kept more than 10

$427 \mathrm{~nm}$ apart by the plasma membrane, we did not observe any Foster resonance energy transfer

428 (FRET), preserving therefore the linearity of the red/green ratio with respect of the amount of

429 cleaved APP (Supplementary Fig. S8). In the other interesting work(Villegas, et al., 2014),

430 APP695 was fused to CFP and YFP at the two opposite terminals, with CFP correctly positioned

431 after the signal peptide, to study the localization of APP fragments and the trafficking processes

432 occurring intracellularly. The authors found that the presence of the tags did not alter the

433 processing of APP by the secretases, and that some intracellular $\mathrm{N}$-terminal fragments - 
434 possibly corresponding to our observed red punctae - do not colocalize with full length APP or

435 C-terminal fragments, in complete agreement with our data. Importantly, the dual tagged APP, 436 at low level of expression, showed a subcellular distribution and a proteolytical profile highly 437 comparable to that of endogenous APP.

438 Interestingly, our results show that cells of the same type (both neuronal and neuroblastoma 439 cells) display different ratios of red/green fluorescence intensity, suggesting that the processing rate of APP is highly variable even within the same population. Furthermore, a certain level of heterogeneity in the red/green intensity ratio is also found within the same cell, indicating that the activity of the proteases can vary in different districts of the cell. This finding is in agreement with previous results showing that there are differences in the processing of APP depending on its targeting on the plasma membrane of highly polarized cells (Haass, et al., 2012) (DeBoer, et al., 2014).

It has been shown that APP can be internalized from the cell surface and specifically targeted to lysosomes(Tang, et al., 2015). Previous works demonstrated that the fluorescence emitted by GFP can be strongly reduced by the acidic $\mathrm{pH}$ of the lysosomal lumen, while the fluorescence emitted by mCherry remains stable in a broad range of pH (Katayama, et al., 2008;Mizushima, Yoshimori and Levine, 2010). These considerations could in part explain the presence in our

451 images of intracellular vesicles with high red fluorescence intensity barely co-localizing with the green fluorescence signal. However, the fact that we did not observe any co-localization with the lysosome marker LAMP1 does not convincingly support this hypothesis. It is rather plausible that the red punctae correspond to cleaved $\mathrm{N}$-terminal fragments transported in vesicles separated from those carrying the full length protein and the C-terminal fragments, as already observed by Villegas et al. (2014).

457 As an alternative to the siRNAs, we have created a variant of mChAPPmGFP carrying a 458 mutation capable of inhibiting the $\alpha$-secretase processing without altering the $\beta$-secretase 
459

460

461

462

463

464

465

466

467

468

469

470

471

472

473

474

475

476

477

478

479

480

481

cleavage. The importance of this construct is the possibility to create transgenic animals, like zebra-fish and rat, which could be used to produce a 3D map of the whole brain showing areas with potentially different $\beta$-secretase activities. Previous data(Sisodia, 1992), together with our confocal microscopy and FACS results, suggest that the P1 mutation makes APP cleavageresistant by a-secretase. However, it is unclear whether it completely inhibits the APP processing. Although cells co-transfected with ADAM10mBFP and $\mathrm{mChAPPP}{ }^{\mathrm{m}} \mathrm{mFP}$ have a cell number in the red/green area ratio similar to that of cells co-expressing mBFP and mChAPPmGFP, the ratio for $\mathrm{mBFP}$ and $\mathrm{mChAPPP}^{1} \mathrm{mGFP}$ transfected cells is much higher than the ratio for $A D A M 10 m B F P$ and $\mathrm{mChAPP}^{\mathrm{P}} \mathrm{mGFP}$ transfected cells. It is possible that under conditions where ADAM10 is strongly overexpressed, a small fraction of $\mathrm{mChAPPP}^{1} \mathrm{mGFP}$ can be still cleaved.

In general, FACS flow cytometry corroborated the reliability of our bioassay and confirmed the heterogeneous processing of APP within the same type of cells. Furthermore, the possibility to separate cells with different rates of APP processing, i.e. different red/green ratios, using the cell-sorting capability of FACS might allow to perform a complete proteomic, lipidomic and transcriptomic analysis of these sub-populations of cells, and to address one of the most puzzling questions in the context of Alzheimer's disease: why the proteolytic processing of APP is higher in certain cells than in others.

477 The combined approach presented in this work could be also used as versatile bioassay to test in living cells the effect of specific molecules on the proteolysis of APP. A more detailed knowledge of the factors governing the processing of APP could contribute to develop alternative drugs for $A D$ treatments. 


\section{Acknowledgements}

483 We thank Dr. Francesco Bemporad, Dr. Daniele Nosi, Dr. Franco Quercioli and Rachele

484 Raggioli for their technical help.

485

486

487

488

489

490

491

492

493

494

495

496

497

498

499

500

501

502

503

504

505

506

507

508 
510

511

512

513

514

515

516

517

518

519

520

521

522

523

524

525

526

527

Bolte, S. \& Cordelieres, F. P. (2006) A guided tour into subcellular colocalization analysis in light microscopy. Journal of microscopy 224, 213-232, doi:10.1111/j.13652818.2006.01706.x.

Collins, A. J., Foley, R. N., Herzog, C., Chavers, B. M., Gilbertson, D., Ishani, A., Kasiske, B. L., Liu, J., Mau, L. W., McBean, M., Murray, A., St Peter, W., Guo, H., Li, Q., Li, S., Li, S., Peng, Y., Qiu, Y., Roberts, T., Skeans, M., Snyder, J., Solid, C., Wang, C., Weinhandl, E., Zaun, D., Arko, C., Chen, S. C., Dalleska, F., Daniels, F., Dunning, S., Ebben, J., Frazier, E., Hanzlik, C., Johnson, R., Sheets, D., Wang, X., Forrest, B., Constantini, E., Everson, S., Eggers, P. W. \& Agodoa, L. (2010) Excerpts from the US Renal Data System 2009 Annual Data Report. American journal of kidney diseases : the official journal of the National Kidney Foundation 55, S1-420, A426-427, doi:10.1053/j.ajkd.2009.10.009.

Costes, S. V., Daelemans, D., Cho, E. H., Dobbin, Z., Pavlakis, G. \& Lockett, S. (2004) Automatic and quantitative measurement of protein-protein colocalization in live cells. Biophysical journal 86, 3993-4003, doi:10.1529/biophysj.103.038422.

Coughlan, K., Huang, X., He, X., Chung, C. H., Li, G. \& Tang, J. (2013) Expression and processing of fluorescent fusion proteins of amyloid precursor protein (APP). Biochimica et biophysica acta 1833, 1562-1571, doi:10.1016/j.bbamcr.2013.03.003.

De Strooper, B. (2010) Proteases and proteolysis in Alzheimer disease: a multifactorial view on the disease process. Physiological reviews 90, 465-494, doi:10.1152/physrev.00023.2009.

DeBoer, S. R., Dolios, G., Wang, R. \& Sisodia, S. S. (2014) Differential release of betaamyloid from dendrite- versus axon-targeted APP. The Journal of neuroscience : the official journal of the Society for Neuroscience 34, 12313-12327, doi:10.1523/JNEUROSCI.2255-14.2014.

Guo, Q., Li, H., Gaddam, S. S., Justice, N. J., Robertson, C. S. \& Zheng, H. (2012) Amyloid precursor protein revisited: neuron-specific expression and highly stable nature of soluble derivatives. The Journal of biological chemistry 287, 2437-2445, doi:10.1074/jbc.M111.315051.

Haass, C. (2004) Take five--BACE and the gamma-secretase quartet conduct Alzheimer's amyloid beta-peptide generation. The EMBO journal 23, 483-488, doi:10.1038/sj.emboj.7600061. 
542
Haass, C., Kaether, C., Thinakaran, G. \& Sisodia, S. (2012) Trafficking and proteolytic processing of APP. Cold Spring Harbor perspectives in medicine 2, a006270, doi:10.1101/cshperspect.a006270.

Howarth, M., Takao, K., Hayashi, Y. \& Ting, A. Y. (2005) Targeting quantum dots to surface proteins in living cells with biotin ligase. Proceedings of the National Academy of Sciences of the United States of America 102, 7583-7588, doi:10.1073/pnas.0503125102.

Katayama, H., Yamamoto, A., Mizushima, N., Yoshimori, T. \& Miyawaki, A. (2008) GFP. like proteins stably accumulate in lysosomes. Cell structure and function 33, 1-12.

Li, Q., Lau, A., Morris, T. J., Guo, L., Fordyce, C. B. \& Stanley, E. F. (2004) A syntaxin 1, Galpha(o), and N-type calcium channel complex at a presynaptic nerve terminal: analysis by quantitative immunocolocalization. The Journal of neuroscience : the official journal of the Society for Neuroscience 24, 4070-4081, doi:10.1523/JNEUROSCI.034604.2004.

MacLeod, R., Hillert, E., Cameron, R. T. \& Baillie, G. S. (2015) The role and therapeutic targeting of $\alpha-, \beta$ - and $\gamma$-secretase in Alzheimer's disease. Future Science O A 1.

Manders, E. M., Stap, J., Brakenhoff, G. J., van Driel, R. \& Aten, J. A. (1992) Dynamics of three-dimensional replication patterns during the S-phase, analysed by double labelling of DNA and confocal microscopy. Journal of cell science 103 ( Pt 3), 857-862.

Mizushima, N., Yoshimori, T. \& Levine, B. (2010) Methods in mammalian autophagy research. Cell 140, 313-326, doi:10.1016/j.cell.2010.01.028.

Nalivaeva, N. N. \& Turner, A. J. (2013) The amyloid precursor protein: a biochemical enigma in brain development, function and disease. FEBS letters 587, 2046-2054, doi:10.1016/j.febslet.2013.05.010.

Schembri, L., Dalibart, R., Tomasello, F., Legembre, P., Ichas, F. \& De Giorgi, F. (2007) The HA tag is cleaved and loses immunoreactivity during apoptosis. Nature methods 4, 107-108, doi:10.1038/nmeth0207-107.

Schneider, C. A., Rasband, W. S. \& Eliceiri, K. W. (2012) NIH Image to ImageJ: 25 years of image analysis. Nature methods 9, 671-675.

Selkoe, D. J. \& Hardy, J. (2016) The amyloid hypothesis of Alzheimer's disease at 25 years. EMBO molecular medicine, doi:10.15252/emmm.201606210. 
573

574

575

576

577

578

579

580

581

582

583

584

585

586

587

588

589

590

591

592

593

594

595

596

597

598

599

600
Senechal, Y., Larmet, Y. \& Dev, K. K. (2006) Unraveling in vivo functions of amyloid precursor protein: insights from knockout and knockdown studies. Neuro-degenerative diseases 3, 134-147, doi:10.1159/000094772.

Shaner, N. C., Campbell, R. E., Steinbach, P. A., Giepmans, B. N., Palmer, A. E. \& Tsien, R. Y. (2004) Improved monomeric red, orange and yellow fluorescent proteins derived from Discosoma sp. red fluorescent protein. Nature biotechnology 22, 15671572, doi:10.1038/nbt1037.

Sisodia, S. S. (1992) Beta-amyloid precursor protein cleavage by a membrane-bound protease. Proceedings of the National Academy of Sciences of the United States of America 89, 6075-6079.

Subach, O. M., Gundorov, I. S., Yoshimura, M., Subach, F. V., Zhang, J., Gruenwald, D., Souslova, E. A., Chudakov, D. M. \& Verkhusha, V. V. (2008) Conversion of red fluorescent protein into a bright blue probe. Chemistry \& biology 15, 1116-1124, doi:10.1016/j.chembiol.2008.08.006.

Subach, O. M., Cranfill, P. J., Davidson, M. W. \& Verkhusha, V. V. (2011) An enhanced monomeric blue fluorescent protein with the high chemical stability of the chromophore. PloS one 6, e28674, doi:10.1371/journal.pone.0028674.

Tang, W., Tam, J. H., Seah, C., Chiu, J., Tyrer, A., Cregan, S. P., Meakin, S. O. \& Pasternak, S. H. (2015) Arf6 controls beta-amyloid production by regulating macropinocytosis of the Amyloid Precursor Protein to lysosomes. Molecular brain 8, 41, doi:10.1186/s13041-015-0129-7.

Villegas, C., Muresan, V. \& Ladescu Muresan, Z. (2014) Dual-tagged amyloid-beta precursor protein reveals distinct transport pathways of its $\mathrm{N}$ - and C-terminal fragments. Human molecular genetics 23, 1631-1643, doi:10.1093/hmg/ddt555.

Zheng, H. \& Koo, E. H. (2011) Biology and pathophysiology of the amyloid precursor protein. Molecular neurodegeneration 6, 27, doi:10.1186/1750-1326-6-27. 


\section{Figure 1}

Schematic representation of the bioassay rationale.

(a) Design of the various chimeric constructs. The N-terminal tags are inserted after the signal peptide (SP) that target APP to the plasma membrane. HA and AP are two short amino acid sequences. HA is recognized by a specific antibody and AP is biotinylated by a specific enzyme. (b) Once mChAPPmGFP is cleaved by $\beta$-secretase, the SAPP $\beta$ fragment carrying mCherry (mCherry-SAPP $\beta$ ) is released into the medium, causing a change in the ratio between the red and green fluorescence intensity. 


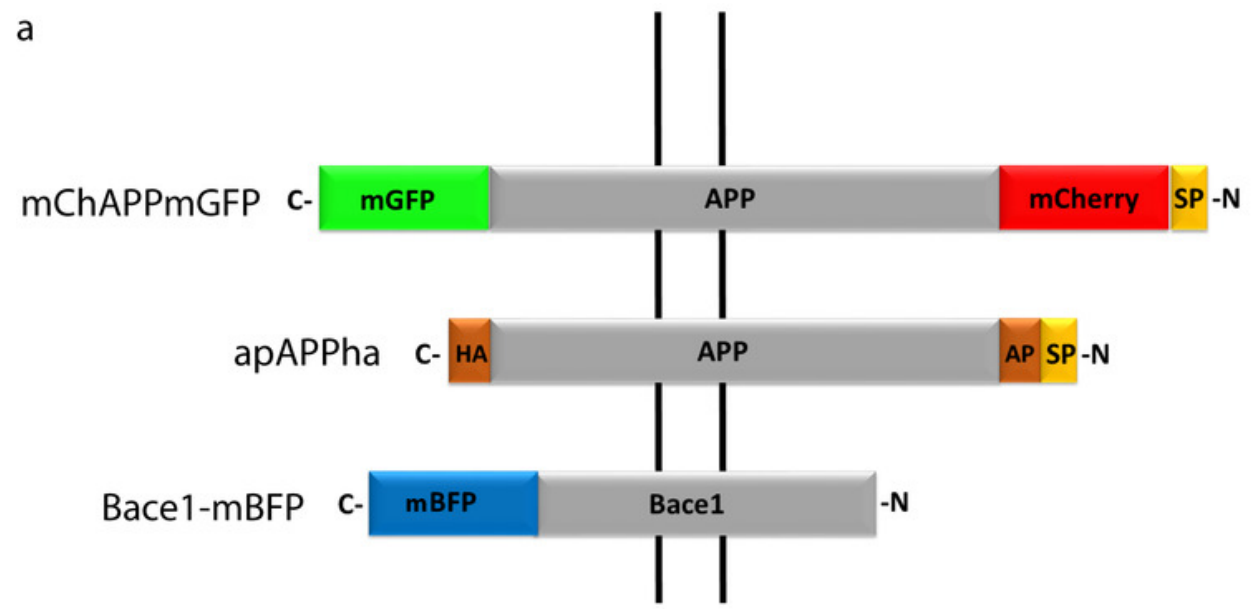

b

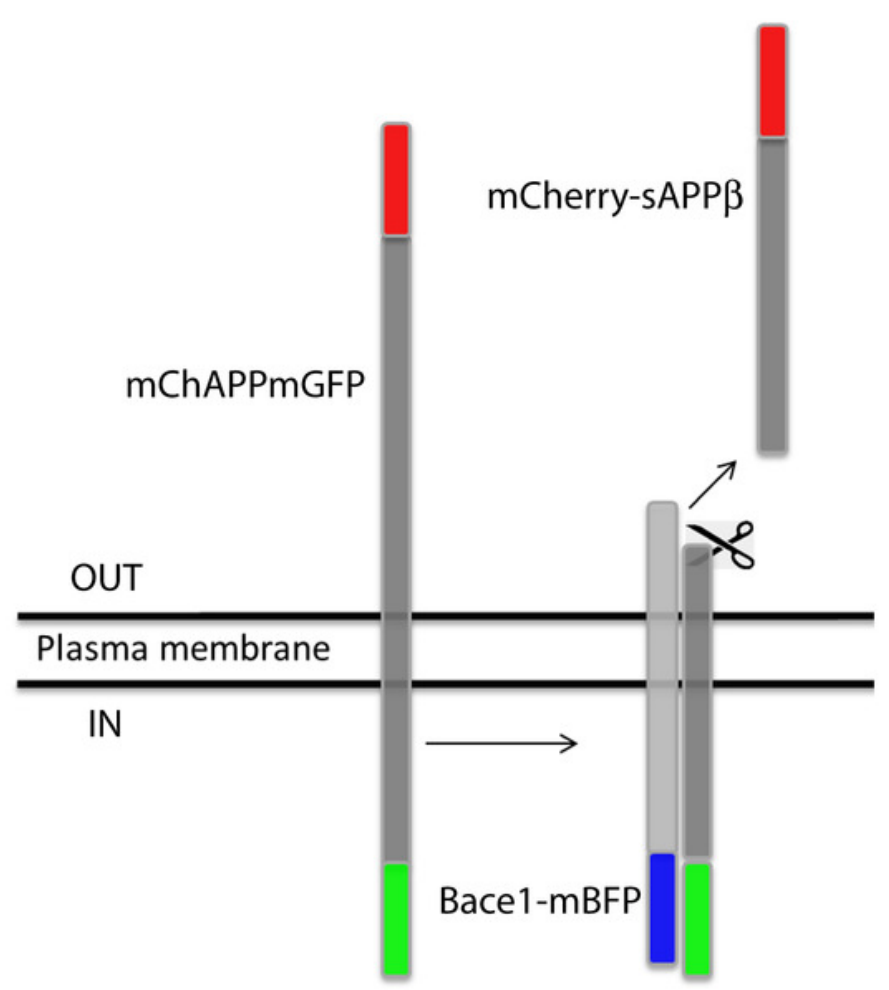




\section{Figure 2}

Correct targeting of mChAPPmGFP and colocalization of mCherry and mGFP.

(a) Maximum intensity projection of a confocal Z-stack of fixed human SH-SY5Y cells transfected with mChAPPmGFP and surface labeled with anti-mCherry coupled to secondary Alexa 405 antibody (blue). Maximum intensity projections of confocal z-stacks of living human SH-SY5Y cells (b) and rat hippocampal neurons (e) transfected with mChAPPmGFP. (h) Maximum intensity projection of a confocal z-stack of fixed and permeabilized SH-SY5Y cells transfected with apAPPha and labeled with streptavidin Alexa 568 (red), which binds to the bionitylated AP tag, and anti-HA coupled to secondary Alexa 488 antibody (green). The high degree of co-localisation of the red and green signals evident from the linear correlation of scatterplots ( $c, f, i)$ and the exponential shape of the Li's intensity correlation analysis ( $d, g, j)$ is confirmed by the Pearson's (Pc) and Manders'coefficients (M1 and M2) close to 1, and by the intensity correlation quotients (ICQ) close to 0.5 . The probability of obtaining the observed Pcs by chance is inversely correlated with the Costes' randomization P value, which is $100 \%$ in all cases. Scalebars, $10 \mu \mathrm{m}$. 

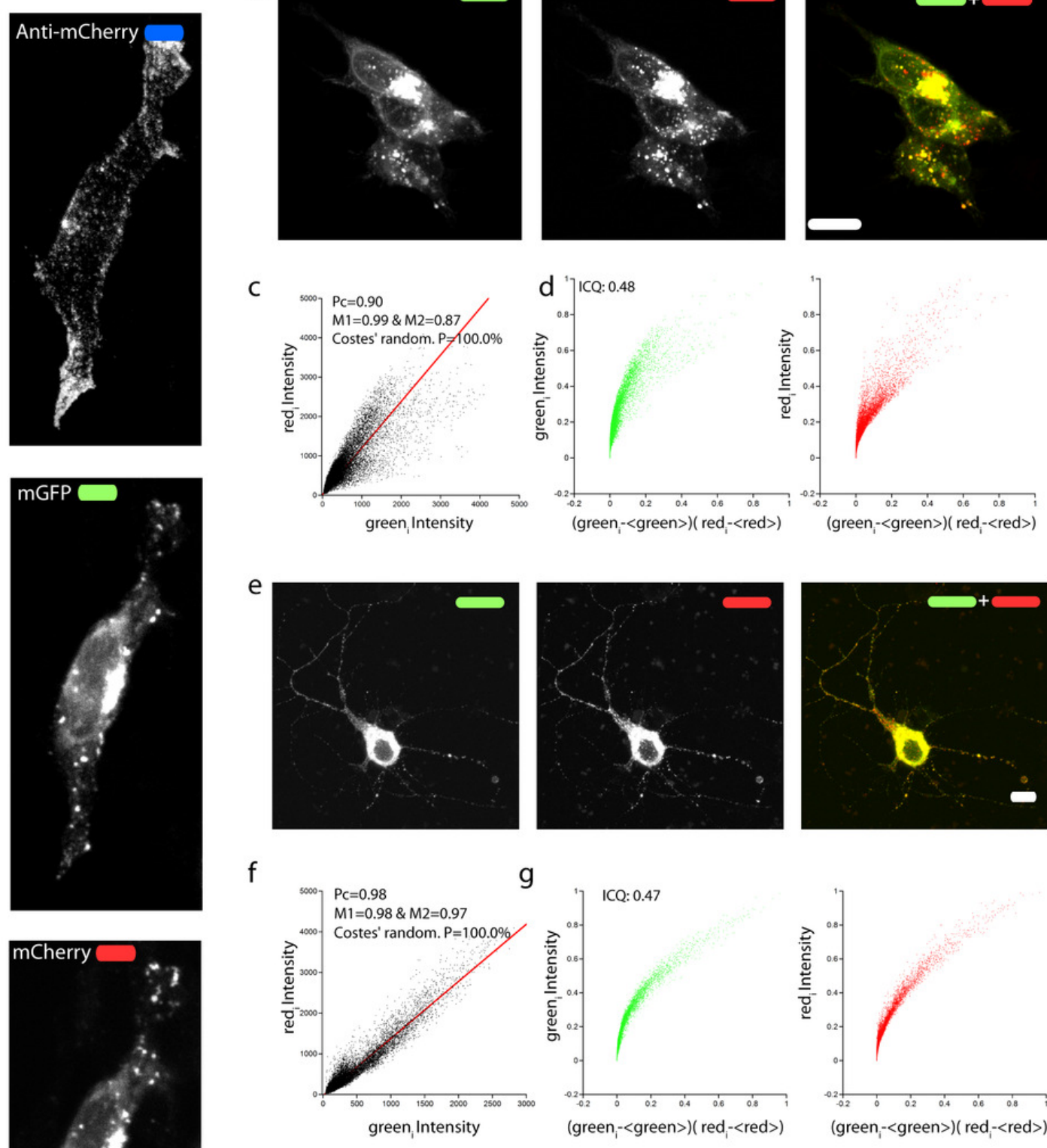

e
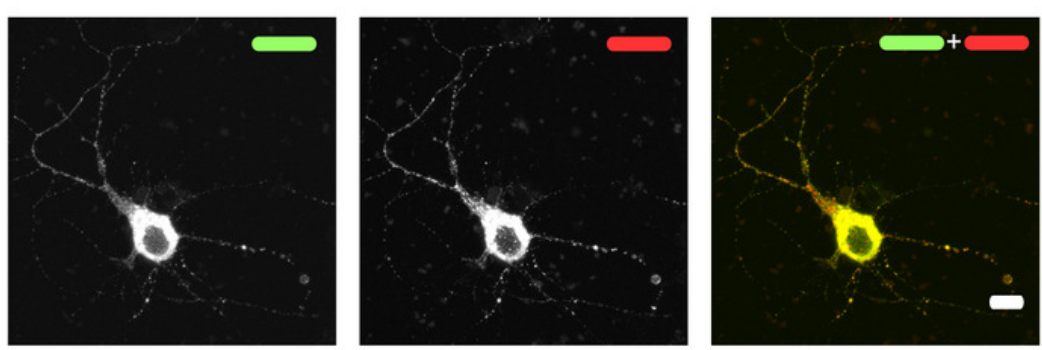

f

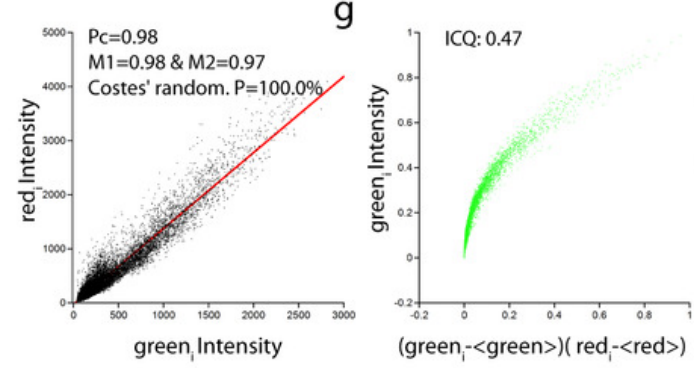

h
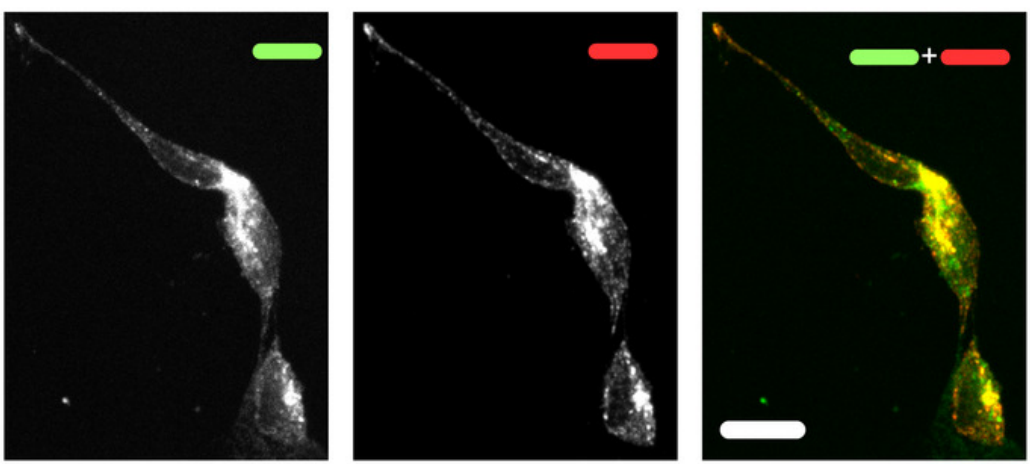

i

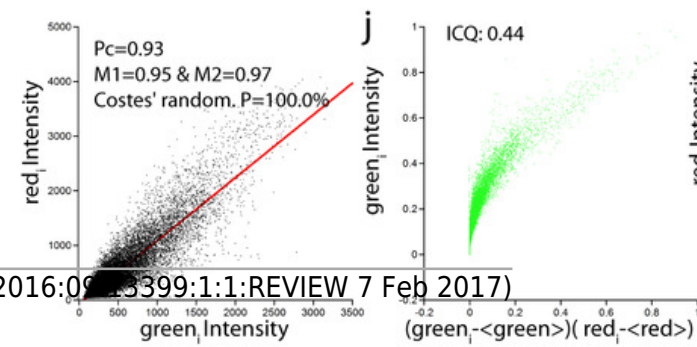

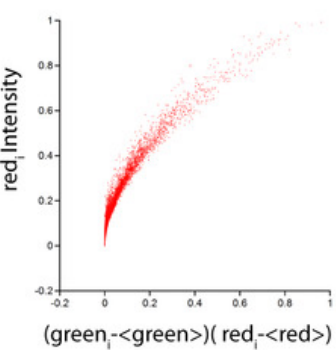

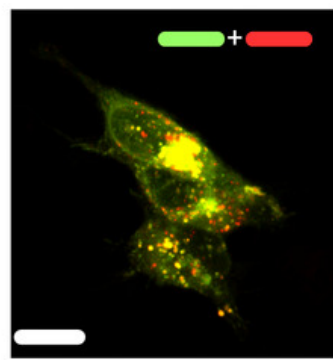

$($ green $-<$ green $>)($ red $-<$ red $>$ )

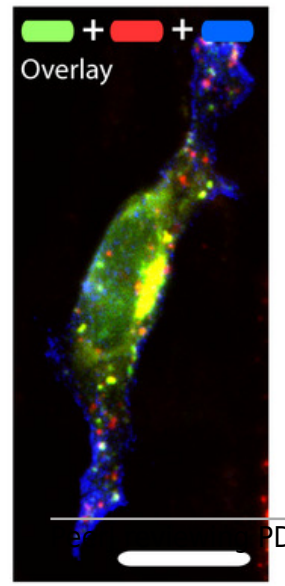

\section{DF}

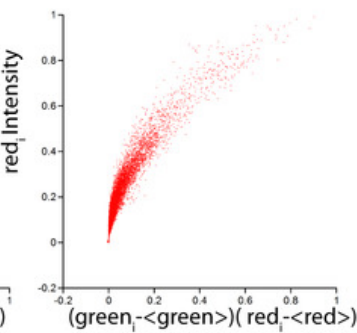




\section{Figure 3}

Red/green fluorescence intensity ratio and intercellular variability of mChAPPmGFP processing.

Maximum intensity projections of confocal z-stacks of living human SH-SY5Y cells (a) and rat hippocampal neurons (b) transfected with mChAPPmGFP. The average red/green fluorescence ratio can fluctuate within the same population of cells (examples indicated by coloured arrows). The red/green ratio image has been mean filtered ( 2 pixels) only for representation purposes. Scalebar $10 \mu \mathrm{m}$. 
a
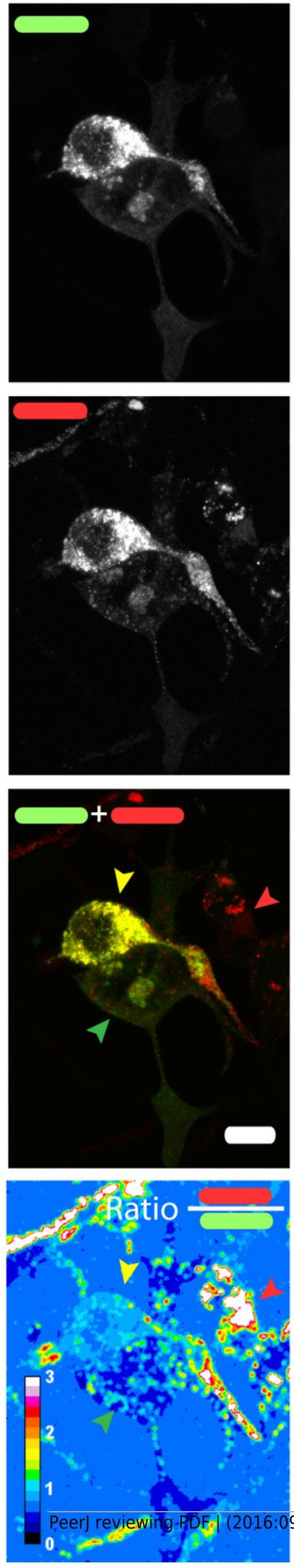

b
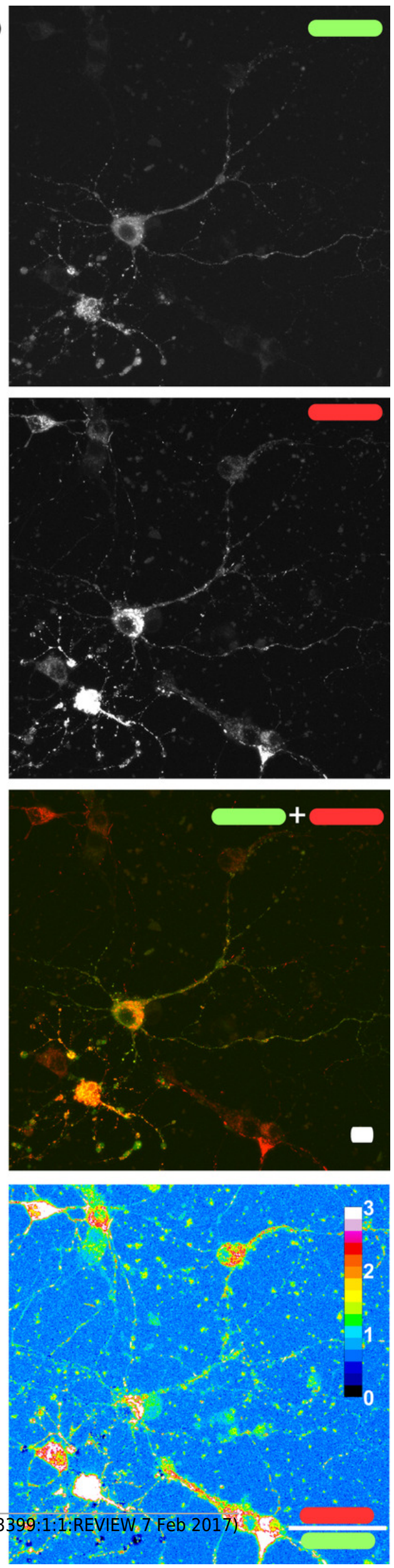


\section{Figure 4}

Proof of principle: overexpressed $\beta$-secretase cleaves efficiently mChAPPmGFP.

(a) Maximum intensity projection of a confocal z-stack of living human SH-SY5Y cells cotransfected with mChAPPmGFP and Bace1-mBFP. The red/green fluorescence ratio image has been mean filtered ( 2 pixels) only for representation purposes. Scalebar, $10 \mu \mathrm{m}$. (b) The different mean red/green fluorescence intensity ratio values of the two cells outlined in ratio image (a) are correlated to the overexpression of Bace1-mBFP (error bars, s.d.).(c) Dot blot of the mean red/green ratio of SH-SY5Y cells under different conditions of transfection. $n>15$ for each condition; $* * *, p<0.001$ according to unpaired Student-t test with unequal variance, run with respect to cells transfected only with mChAPPMGFP or mChAPP770mGFP. (d) Superimposed westernblots of transfected SH-SY5Y cell lysates (magenta) and extracellular media (cyan), labelled with anti-mCherry. The lysate blot was repeatedly stripped and reprobed with anti-GFP, anti-APP and anti-A 3 . The lower band corresponds to mCherry-SAPP, while the higher to full-length mChAPPmGFP. (e) The extracellular medium of SH-SY5Y cells under different conditions of transfection was analysed with a fluorescence spectrophotometer. Emission spectra were collected following excitation at $550 \mathrm{~nm}$. 

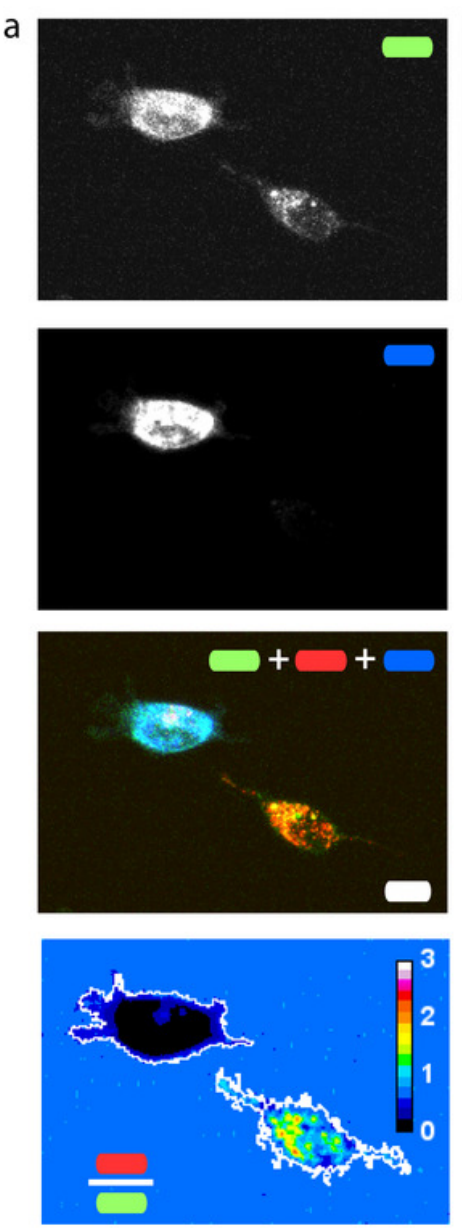

c

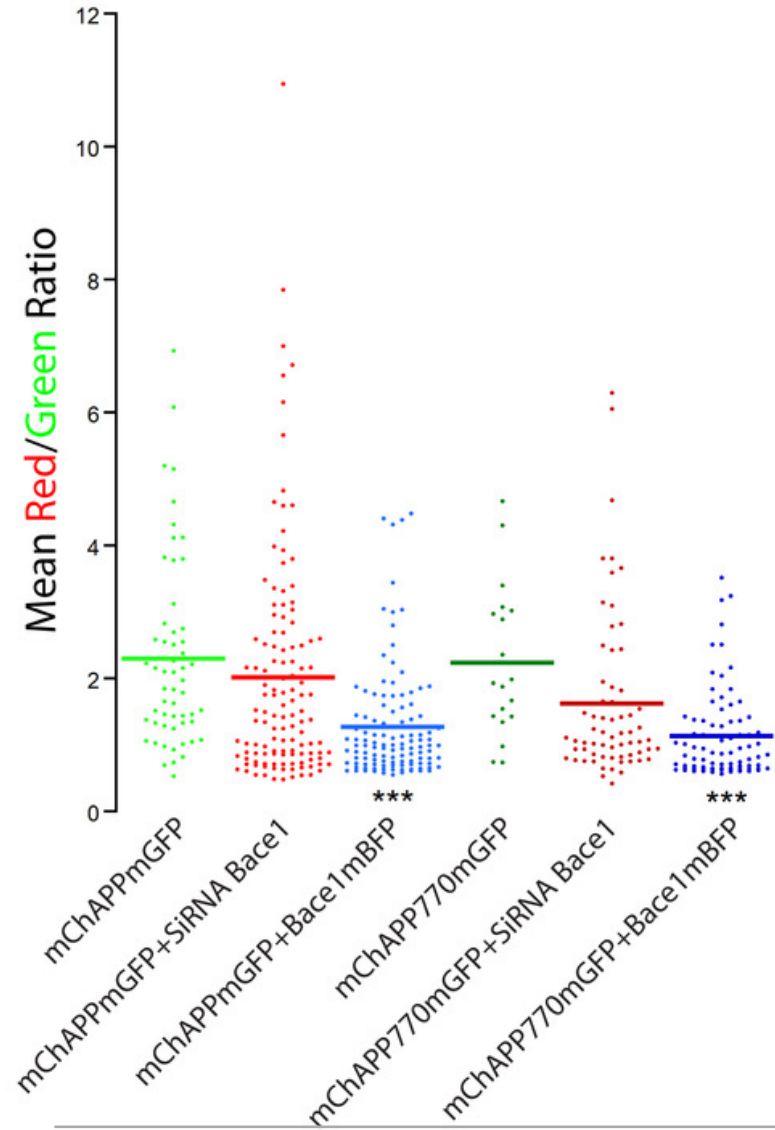

Peer) reviewing PDF | (2016:09:13399:1:1:REVIEW 7 Feb 2017)

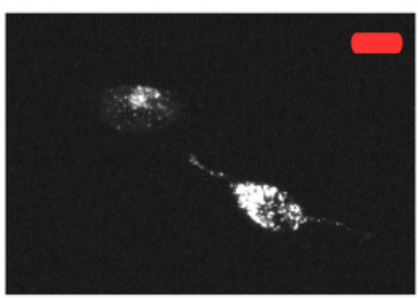

$b$

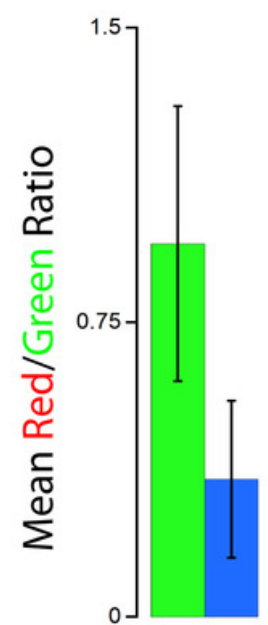

d

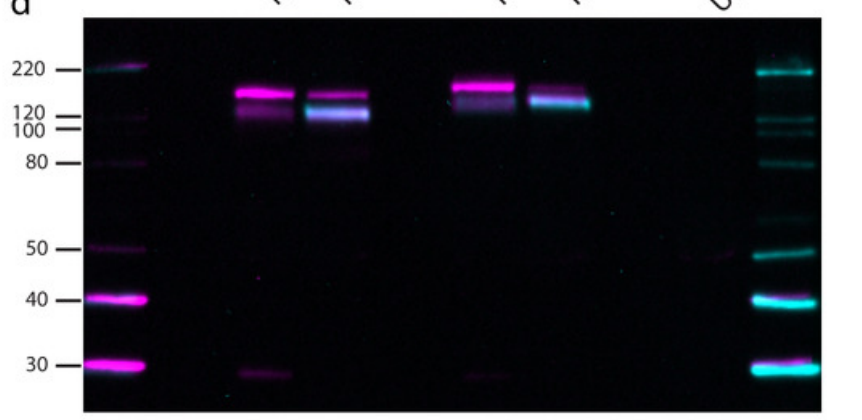

Anti-mCherry Supernatant/Pellet

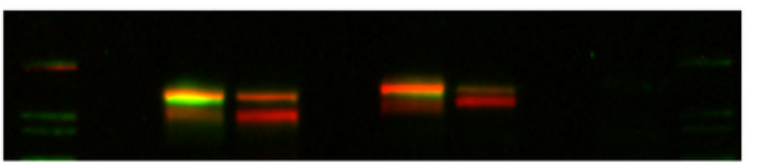

Pellet anti-mCherry/anti-GFP

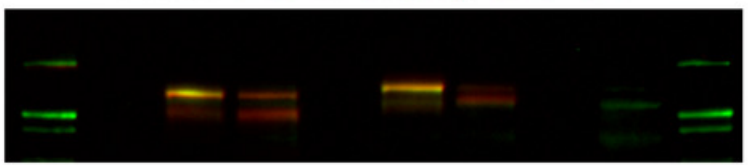

Pellet anti-mCherry/anti-APP

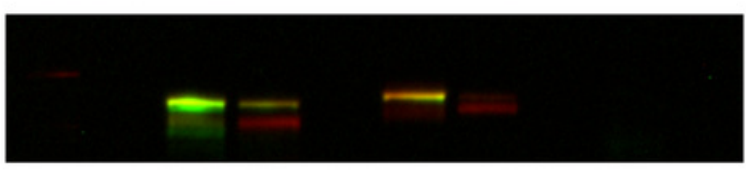

Pellet anti-mCherry/anti-A $\beta$

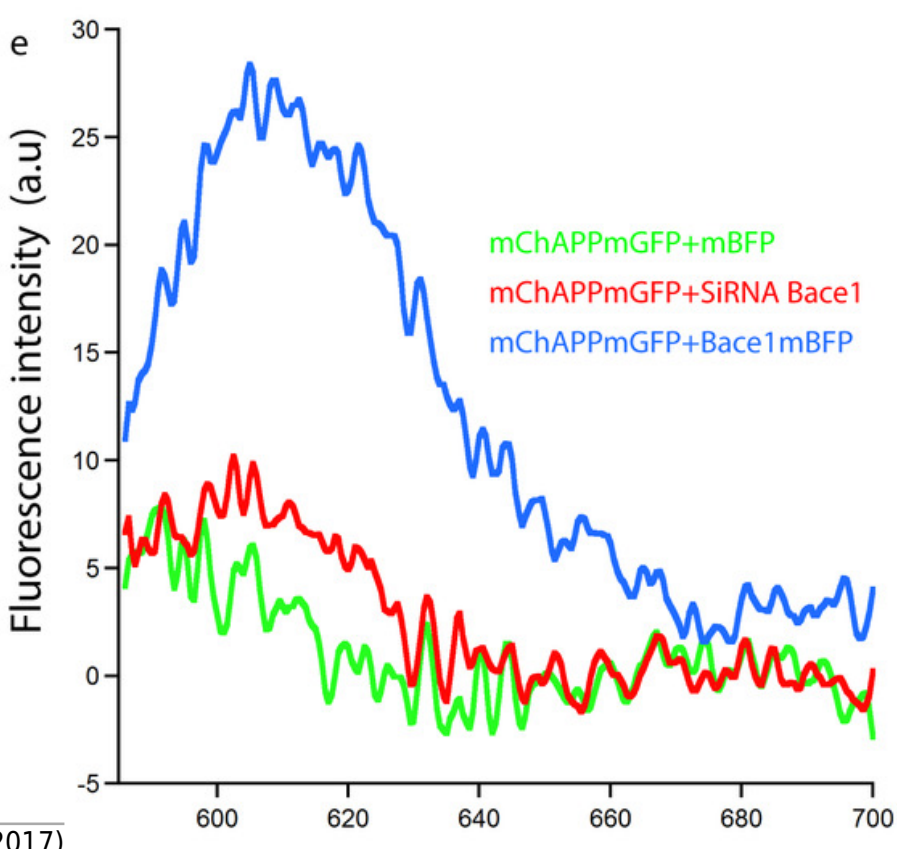

Emission wavelnegth $(\mathrm{nm})$ 


\section{Figure 5}

mChAPPmGFP is properly processed in neurons.

Maximum intensity projections of confocal z-stacks of living rat hippocampal neurons cotransfected with mChAPPmGFP and mBFP (a) or Bace1-mBFP (b). The red/green ratio images were mean filtered ( 2 pixels) only for representation purposes. The insets correspond to the magnified regions outlined in the ratio images. Scalebars $10 \mu \mathrm{m}$. 

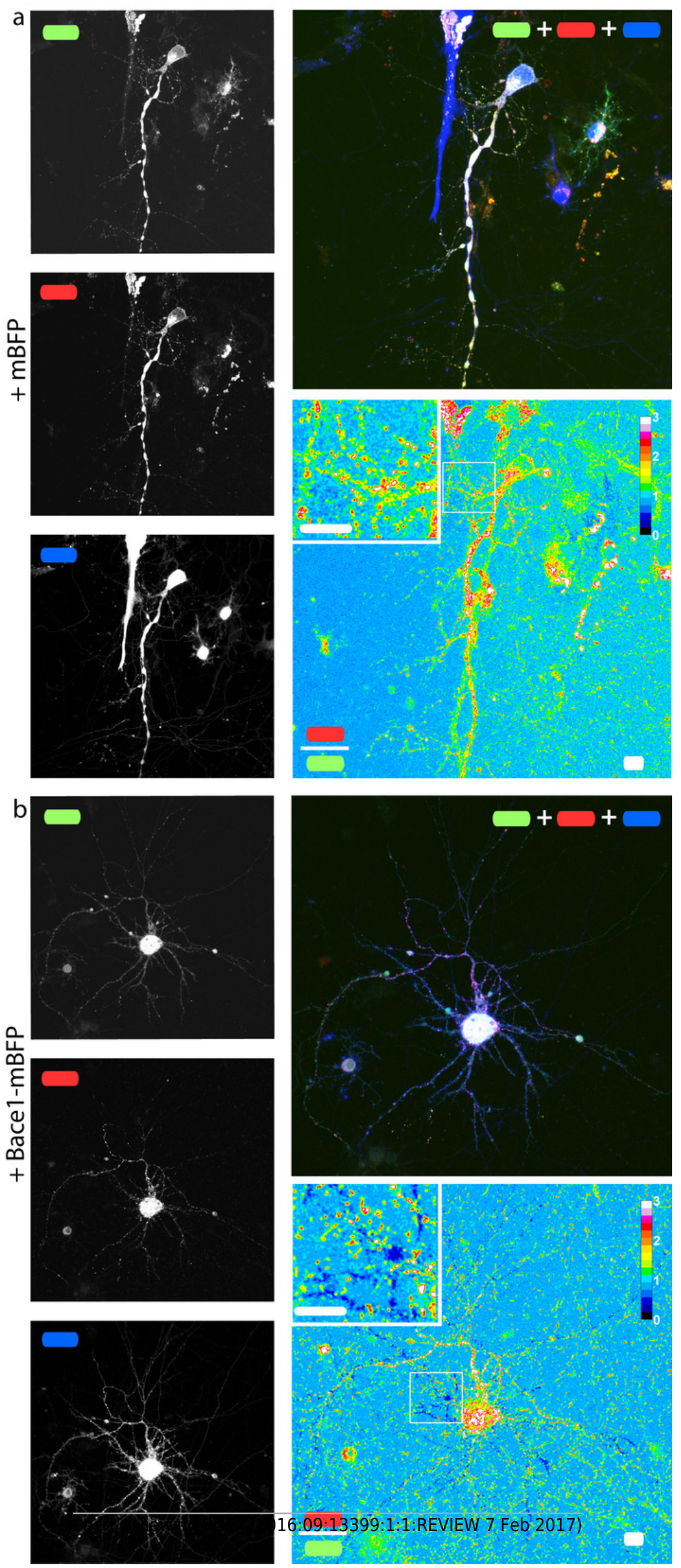


\section{Figure 6}

FACS flowcitometry as additional way to screen mChAPPmGFP processing.

(a) Each dot in the scatter plot represents the green and red fluorescence intensity of a single cell. The two different areas (red and green) in the scatter plot were arbitrarily chosen so that both areas contained the same number of SH-SY5Y cells co-transfected with mChaPPmGFP and control siRNA. These regions were used to gate the scatter plots of cells co-transfected with mChAPPmGFP and Bace1-mBFP, Bace1-siRNA, or control siRNA, and calculate the mean ratio between the number of cells in the red area over the number of cells in the green area (b). $n=3$ independent experiments; error bars, s.d.; $p<0.0001$ according to unpaired Student-t test, run with respect to cells transfected with mChAPPmGFP and control siRNA. 

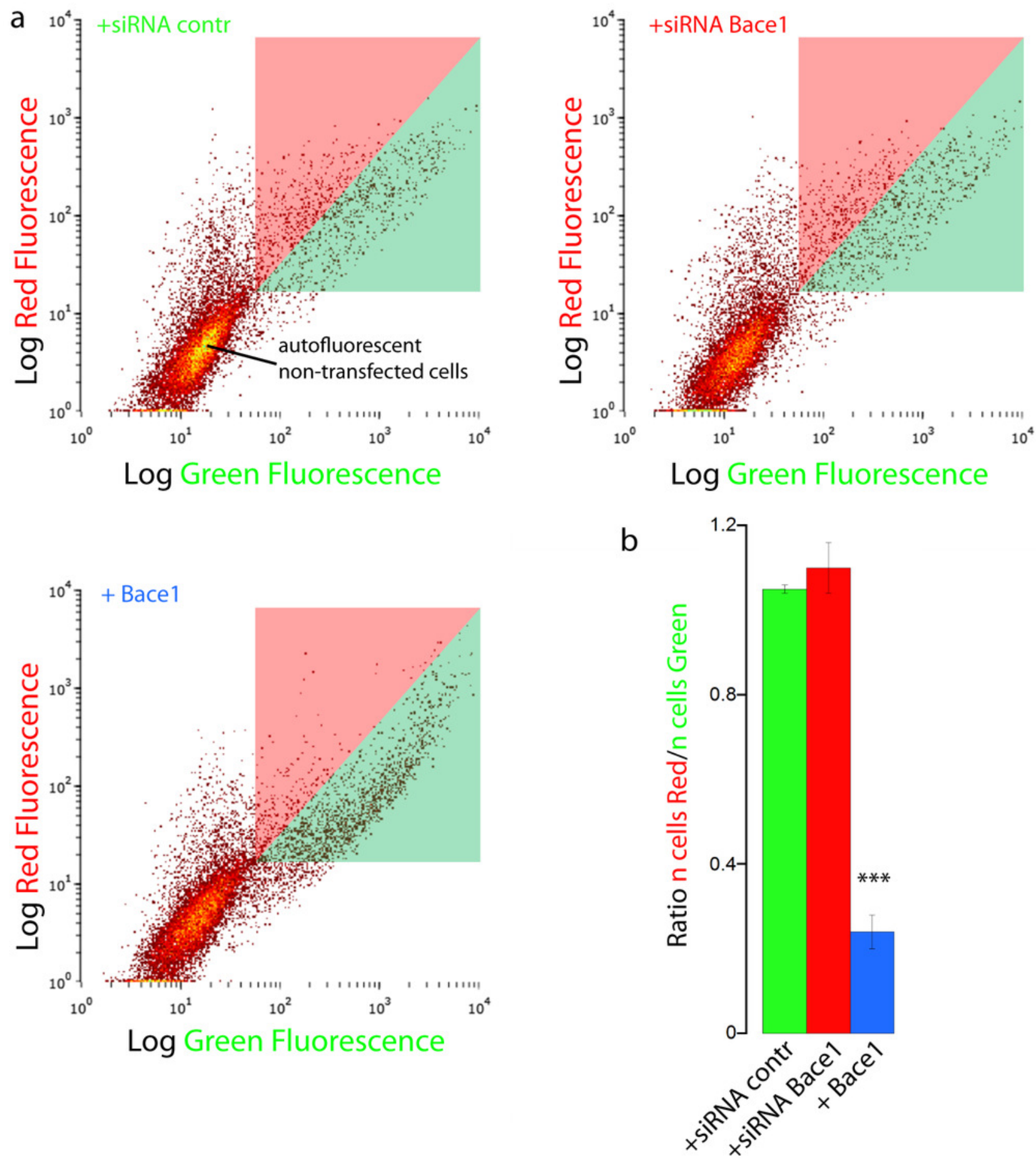


\section{Figure 7}

The mChAPPP1 mGFP mutant is not efficiently cleaved by the $\alpha$-secretase ADAM10.

(a) Maximum intensity projection of confocal z-stacks of living human SH-SY5Y cells under different conditions of transfection. The red/green ratio images were mean filtered ( 2 pixels) only for representation purposes. Scalebar $10 \mu \mathrm{m}$. (b) Dot blot of the mean red/green ratio of SH-SY5Y cells under different conditions of transfection. $n \geq 10$ for each condition; ***, $p<0.001$ according to unpaired Student-t test with unequal variance. (c) FACS flowcytometry confirms the inhibitory effect of the P1 mutation on $\alpha$-secretase, but not $\beta$-secretase, processing. $\mathrm{n}=3$ independent experiments; error bars, s.d.; $*, \mathrm{p}<0.05, * * *, \mathrm{p}<0.001$, according to unpaired Student-t test with unequal variance. Scatterplots from one experiment are reported in Supplementary Figure S7. 

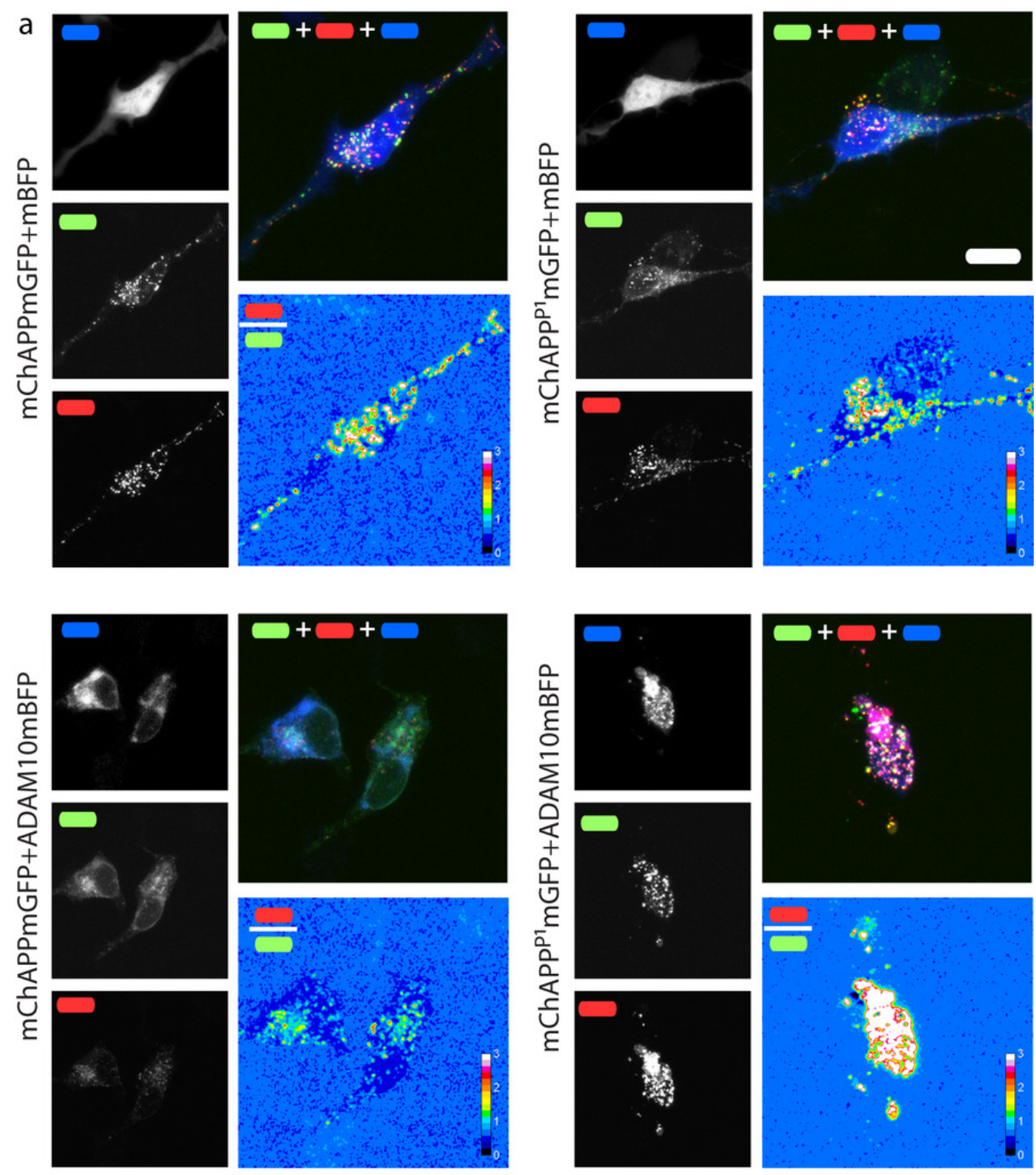

b
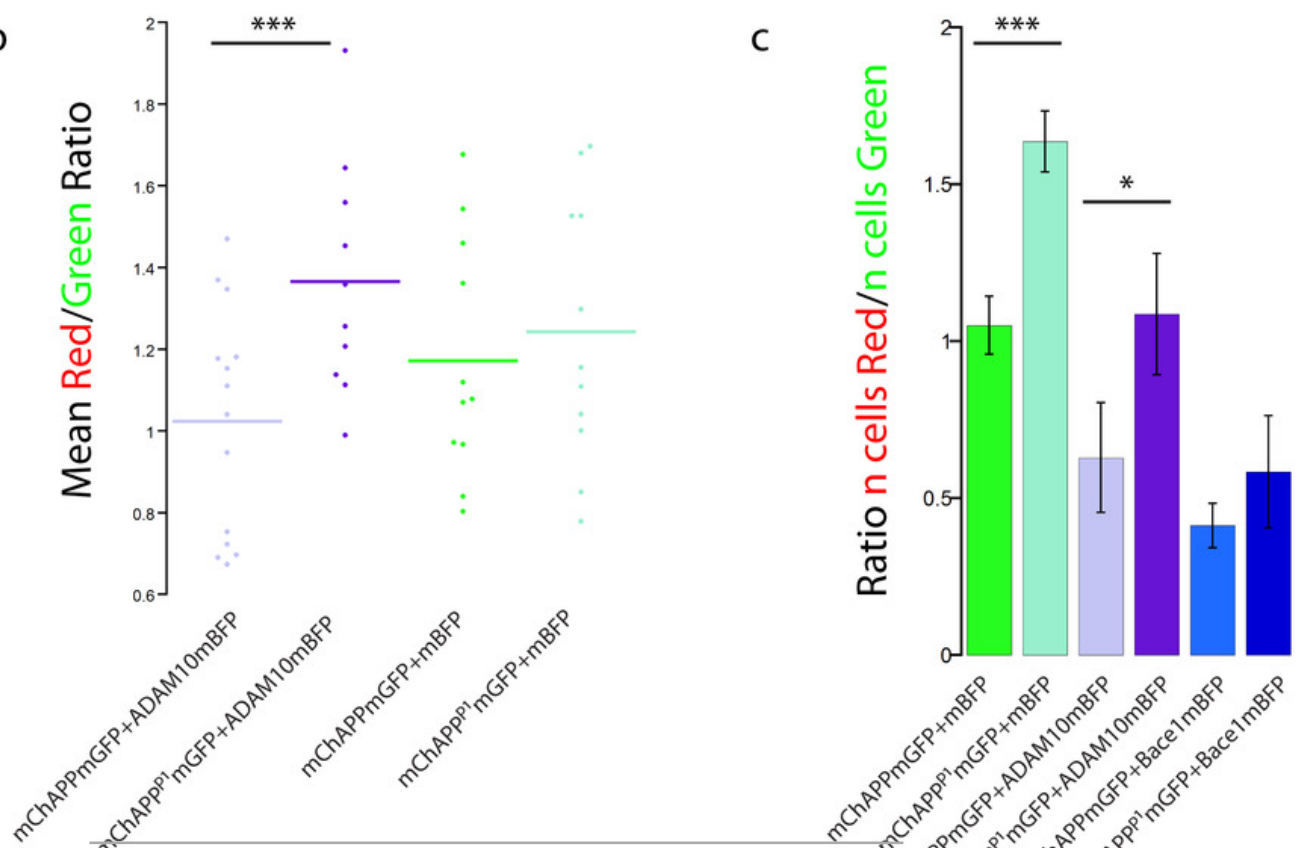